\title{
Quantifying the incremental cost of complications associated with mitral valve surgery in the United States
}

\author{
Alexander Iribarne, MD, MS, ${ }^{\mathrm{a}}$ John D. Burgener, MBA, MS, ${ }^{\mathrm{b}}$ Kimberly Hong, MHSA, ${ }^{\mathrm{a}}$ \\ Jai Raman, MD, PhD, ${ }^{\mathrm{c}}$ Shahab Akhter, MD, ${ }^{\mathrm{c}}$ Rachel Easterwood, BA, ${ }^{\mathrm{a}}$ Valluvan Jeevanandam, MD, ${ }^{\mathrm{c}}$ and \\ Mark J. Russo, MD, MS ${ }^{\mathrm{c}, \mathrm{d}}$
}

Objective: The goal of this study was to quantify the net increase in resource use associated with complications after isolated mitral valve surgery.

\begin{abstract}
Methods: Deidentified patient-level claims data on a random sample of mitral valve operations performed in the United States from January 1, 2006, to December 31, 2007, were obtained from the National Inpatient Sample $(n=16,788)$. Patients with major concomitant cardiac procedures were excluded from the analysis for a net sample size of 6297 patients. Risk-adjusted median total hospital costs and length of stay were analyzed by major complications, including pneumonia, sepsis, stroke, renal failure requiring hemodialysis, cardiac tamponade, myocardial infarction, gastrointestinal bleed, and venous thromboembolism.
\end{abstract}

\begin{abstract}
Results: There were a total of 1323 complication events that occurred in 1089 patients. The most common complication was pneumonia $(\mathrm{n}=346,5.5 \%)$, which was associated with a $\$ 29,692$ increase in hospital costs and a 10.2-day increase in median length of stay $(P<.001)$. The most costly complication was cardiac tamponade, which resulted in an increase in hospital cost of $\$ 56,547$ and an increase in length of stay of 19.3 days $(P<.001)$. There was a stepwise association between the hospital costs and length of stay and the number of complications per patient $(P<.001)$. There was also a significant association between the discharge location and the occurrence of a complication, with $25 \%$ more patients who underwent routine home discharge when there were no complications $(P<.001)$.
\end{abstract}

Conclusions: In patients undergoing isolated mitral valve surgery, postoperative complications were associated with significant increases in mortality, hospital costs, and length of stay, as well as with discharge location. With growing national attention to improving quality and containing costs, it is important to understand the nature and impact of complications on outcomes and costs. (J Thorac Cardiovasc Surg 2012;143:864-72)

Among patients undergoing mitral valve surgery (MVS), significant improvements in outcomes have been observed nationally over the past decade. ${ }^{1,2}$ Data from large national registries have demonstrated that length of stay and perioperative morbidity and mortality have achieved acceptably low levels. ${ }^{3,4}$ These improvements in outcomes have occurred in the face of an increased frequency of comorbidities among patients presenting for MVS, including an increased prevalence of diabetes, hypertension, and hyperlipidemia. ${ }^{5}$ Despite advances in care, with an aging

From the Division of Cardiothoracic Surgery, ${ }^{\text {a }}$ Department of Surgery, College of Physicians and Surgeons, Columbia University, New York, NY; The Oryx Group, ${ }^{b}$ Miami, Fla; Section of Cardiac and Thoracic Surgery, ${ }^{\mathrm{c}}$ University of Chicago Medical Center, Chicago, Ill; and Center for Health and the Social Sciences, ${ }^{d}$ University of Chicago, Chicago, Ill

Disclosures: Authors have nothing to disclose with regard to commercial support.

Read at the 37th Annual Meeting of The Western Thoracic Surgical Association, Colorado Springs, Colorado, June 22-25, 2011.

Received for publication June 20, 2011; revisions received Dec 12, 2011; accepted for publication Jan 6, 2012.

Address for reprints: Mark J. Russo, MD, MS, Section of Cardiac and Thoracic Surgery, University of Chicago Medical Center, 5841 S. Maryland Avenue, MC 5040, Chicago, IL 60637 (E-mail: mrusso@surgery.bsd.uchicago.edu).

$0022-5223 / \$ 36.00$

Copyright (c) 2012 by The American Association for Thoracic Surgery doi:10.1016/j.jtcvs.2012.01.032
US population, patients will continue to face significant perioperative risk after MVS.

It is well recognized that postoperative complications are associated with increased mortality, prolonged hospital stay, and greater resource use. ${ }^{6}$ However, detailed data regarding the clinical and economic impact of postoperative complications, particularly after valvular surgery, remain scarce. $^{7}$ Availability of this information could be used to target further quality improvement efforts, which may significantly improve outcomes for patients and costcontainment efforts in an era when cost-effectiveness is at the forefront of healthcare policy initiatives. ${ }^{8}$

The purpose of this study was to characterize the impact of postoperative complications on clinical outcomes, such as mortality and discharge disposition, and the net increase in resource use, including in-hospital cost and length of stay, in a national cohort of patients undergoing isolated MVS.

\section{METHODS AND MATERIALS}

Data Source and Study Population

The Nationwide Inpatient Sample (NIS), which is sponsored by the Agency for Healthcare Research and Quality, Healthcare Cost and Utilization Project, was used to identify patient discharges related to MVS that 


\section{Abbreviations and Acronyms \\ AVR = aortic valve replacement \\ $\mathrm{CABG}=$ coronary artery bypass grafting \\ CI = confidence interval \\ CMS $=$ Centers for Medicaid and Medicare Services \\ ICD-9-CM = International Classification of Diseases, Ninth Revision, Clinical Modification \\ MVR = mitral valve replacement \\ MVS = mitral valve surgery \\ NIS $=$ Nationwide Inpatient Sample \\ OR $=$ odds ratio \\ STS $=$ Society of Thoracic Surgeons}

occurred between January 1, 2006, and December 31, $2007(\mathrm{n}=16,788)$. The NIS provides only deidentified patient claims data, and thus this analysis qualified for institutional review board exception. The NIS is a $20 \%$ sampling of abstracted discharge data from a national survey of all nonfederal acute care hospitals in the United States and contains discharge records from more than 1000 hospitals in 37 states. $^{9}$

The NIS contains up to 15 procedure codes per patient using the International Classification of Diseases, Ninth Revision, Clinical Modification (ICD-9-CM) procedure code index. MVS was abstracted using ICD-9CM codes $35.23,35.24$, and 35.12 in the first, second, or third procedure code position. For the primary analysis, major concomitant cardiac procedures (coronary artery bypass grafting [CABG], aortic valve surgery, tricuspid valve surgery, and arrhythmia surgery) were excluded using appropriate ICD-9-CM codes to study only patients with isolated MVS $(n=6297)$ and avoid the potential confounding effect of multiple procedures on resource use. In addition, patients aged less than 18 years were excluded from the analysis. The NIS does not provide data on repeat procedures.

\section{Clinical Outcomes and Complications}

Major clinical outcomes of interest included length of hospitalization, in-hospital morbidity, and in-hospital mortality. Given that the NIS includes data on up to 15 diagnoses per discharge, a focus on specific major postoperative complications (eg, pneumonia) rather than complication systems-based categories (eg, respiratory complications) was used to maximize specificity of results. Specific major postoperative complications extracted from the NIS using appropriate ICD-9 codes included pneumonia (ICD-9-CM codes 480-487); acute renal failure (ICD-9-CM code 584); septicemia (ICD-9-CM code 038); acute myocardial infarction (ICD-9CM code 410); stroke (ICD-9-CM code 436.* and 437.*); cardiac tamponade (ICD-9-CM code 423.3); gastrointestinal bleed (ICD-9-CM codes 530.82, 535.61 and 578.9); and venous thromboembolism (ICD-9-CM codes $451 .^{*}$ and $\left.453 .^{*}\right)$. The number of complications per patient was also analyzed to study the multiplicative effect of complications on resource use. Last, discharge location was assessed for all patients.

\section{Cost Outcomes}

Total billed charges for each hospitalization are present in the NIS dataset. These data reflect the amount hospitals billed for services rendered rather than the costs for the specific hospitalization or the amount hospitals received in payments. Estimated institutional cost data were obtained by multiplying Healthcare Cost and Utilization Project supplied cost-tocharge ratios by total charges. Grouped average cost-to-charge ratios are a weighted average for the hospitals in the group (defined by state, urban/rural, investor owned/other, and number of beds) and use the proportion of group beds as the weight for each hospital. Total costs related to the complication were calculated on the basis of incremental cost multiplied by the annual incidence multiplied by a factor of 5 to account for the $20 \%$ sample. All costs were rounded to the nearest $\$ 100$.

\section{Statistical Analysis of Cost and Clinical End Points}

Because medical costs are traditionally right skewed, as costs cannot be negative, we report median cost in the analysis unless otherwise specified. For univariate analysis of median cost, results of the Wilcoxon rank-sum test are reported. To assess the independent effect of complications on total median hospital costs, multivariable quantile regression using 1000 bootstrapped iterations was used where the outcome of interest was median cost. Demographic variables used in multivariable regression included age and all comorbidities presented in Appendix 1 that had a $P$ value less than .20 in univariate analysis.

For clinical data, continuous variables were reported as mean \pm standard error and compared using the Student $t$ test or Wilcoxon rank-sum test when noted. Categoric variables were reported as percentages and compared using the chi-square or Fisher exact test when appropriate. Median length of stay was reported rather than mean given the potential for outliers, and risk-adjusted length of stay was analyzed in a similar fashion as cost using multivariable quantile regression with 1000 bootstrapped iterations. Long-term survival data are not present in the current dataset; however, in-hospital mortality is coded and reported. Logistic regression was used to assess the independent effect of the number of complications on in-hospital mortality. All reported $P$ values are 2-sided. All statistical analyses were performed using Stata 11 (StataCorp, College Station, Tex)

\section{RESULTS}

\section{Study Population}

From January 1, 2006, to December 31, 2007, there were 16,788 patients in the NIS registry who underwent MVS. Appendix 2 shows the distribution of repairs, replacements, and concomitant procedures in the series. There were 6297 $(37.5 \%)$ isolated mitral valve operations that formed the study cohort for the primary end points. Among isolated mitral valve operations, cases were distributed as follows: mechanical mitral valve replacement $(27.5 \%, \mathrm{n}=1732)$, tissue mitral valve replacement $(20.4 \%, \mathrm{n}=1282)$, and mitral valve repair $(52.1 \%, \mathrm{n}=3283)$. The distribution of baseline characteristics of the study cohort is summarized in Table 1. The mean age of patients was $64.6 \pm 13.4$ years, the majority of procedures were elective $(65.2 \%$, $\mathrm{n}=4103$ ), and the 2 most common comorbidities included peripheral vascular disease $(38.2 \%, \mathrm{n}=2406)$ and heart failure $(38 \%, \mathrm{n}=2396)$.

\section{Clinical Outcomes}

The mean and median lengths of stay were $11.6 \pm 12.1$ and 8 days, respectively, for all isolated mitral valve operations. The distribution of postoperative complications for the series is summarized in Table 2. There were a total of 1323 complication events that occurred in 1089 patients. The 3 most common complications were pneumonia $(5.5 \%, \mathrm{n}=346)$, sepsis $(4.1 \%, \mathrm{n}=261)$, and renal failure requiring dialysis $(3.5 \%, \mathrm{n}=223)$. When analyzed by the 
TABLE 1. Baseline demographics and comorbidities of study cohort

\begin{tabular}{lcc}
\hline \multicolumn{1}{c}{ Baseline variable } & $\begin{array}{c}\text { Mean or } \\
\text { frequency }\end{array}$ & $\begin{array}{c}\text { Standard } \\
\text { error or } \%\end{array}$ \\
\hline Demographics & & \\
Age, y & 64.6 & 13.4 \\
Male & 3062 & 48.6 \\
Elective procedure & 4103 & 65.2 \\
Primary payer: Medicare & 2781 & 44.2 \\
Primary payer: Private & 2558 & 40.6 \\
Comorbidities & & \\
Peripheral vascular disease & 2406 & 38.2 \\
Heart failure & 2396 & 38.0 \\
Chronic obstructive pulmonary disease & 1064 & 16.9 \\
History of any malignancy & 946 & 15.0 \\
Diabetes mellitus & 670 & 10.6 \\
Chronic kidney disease & 571 & 9.1 \\
Cerebrovascular disease & 377 & 6.0 \\
Previous myocardial infarction & 219 & 3.5 \\
Rheumatologic disease & 142 & 2.3 \\
Diabetes with chronic complications & 82 & 1.3 \\
Moderate or severe liver dysfunction & 82 & 1.3 \\
Peptic ulcer disease & 45 & 0.7 \\
Mild liver dysfunction & 30 & 0.5 \\
Hemiplegia or paraplegia & 23 & 0.4 \\
Metastatic solid tumor & 14 & 0.2 \\
Human immunodeficiency virus & 13 & 0.2 \\
Dementia & 2 & 0.0 \\
\hline
\end{tabular}

number of complications per patient, $14.1 \%(\mathrm{n}=887)$ of patients had 1 complication and $2.7 \%(\mathrm{n}=171)$ had 2 complications. In-hospital mortality for the series was $4.0 \%$ $(\mathrm{n}=253)$; however, among elective patients, the mortality rate was $2.3 \%$. The 2 most common discharge locations were routine home discharge $(48.1 \%, \mathrm{n}=3027)$ and home with home health care $(32.1 \%, \mathrm{n}=2024)$. There was a risk-adjusted stepwise increase in the probability of in-hospital mortality for 1 (odds ratio [OR], 3.8; confidence interval [CI], 2.9-5.3, $P<.001$ ), versus 2 complications (OR, 8.5; CI, 5.4-13.4, $P<.001$ ), versus 3 complications (OR, 9.9; CI, 3.8-26.1, $P<.001$ ) when compared with no complications as the reference group.

\section{Resource Use}

The mean and median cost of hospitalization for the series was $\$ 46,229 \pm \$ 37,193$ and $\$ 35,446$, respectively. The complications and associated risk-adjusted net increase in median length of stay and risk-adjusted net increase in median total hospital cost are summarized in Table 3. Cardiac tamponade was both the most costly complication, resulting in a median net increase in hospital costs of $\$ 56,547$ $(P<.001)$, and the complication resulting in the largest net increase in hospital stay at 19.3 days $(P<.001)$. Of note, cardiac tamponade was the least frequent complication in the series, occurring in $0.1 \%$ of patients $(n=8)$. The
TABLE 2. Distribution of in-hospital complication events and number of complications per patient in addition to discharge disposition

\begin{tabular}{lcc}
\hline & Frequency & $\%$ \\
\hline Morbidity events* & & \\
Pneumonia & 346 & 5.5 \\
Sepsis & 261 & 4.1 \\
Renal failure requiring hemodialysis & 223 & 3.5 \\
Acute myocardial infarction & 172 & 2.7 \\
Stroke & 144 & 2.3 \\
Gastrointestinal bleed & 120 & 1.9 \\
Venous thromboembolism & 49 & 0.8 \\
Cardiac tamponade & 8 & 0.1 \\
Complications per patient & & \\
0 & 5208 & 82.7 \\
1 & 887 & 14.1 \\
2 & 171 & 2.7 \\
3 & 30 & 0.48 \\
4 & 1 & 0.02 \\
Mortality & & \\
In-hospital mortality & 253 & 4.0 \\
Discharge location & & \\
Routine discharge & 3027 & 48.1 \\
Home health care & 2024 & 32.1 \\
SNF, ICF, other & 929 & 14.8 \\
Died & 253 & 4.0 \\
Transfer to short-term hospital & 36 & 0.6 \\
Unknown & 23 & 0.4 \\
Against medical advice & 5 & 0.08 \\
\hline SNF, Skilled nursing facility; $I C F$, intermediate care facility. $*$ Morbidity events reflect \\
all occurrences of such events in the study cohort rather than the number of events per \\
patient. & & \\
& &
\end{tabular}

most common complication in the series was pneumonia $(5.5 \%, \mathrm{n}=346)$, and this complication also resulted in the second largest increase in median net hospital costs $(\$ 29,692, P<.001)$ and the second largest increase in length of hospitalization (10.2 days, $P<.001$ ). Moreover, pneumonia resulted in the single greatest total incremental annual cost at between $\$ 23.9$ and $\$ 27.5$ million. Sepsis and renal failure requiring dialysis resulted in the second and third greatest total incremental costs. Given the significant resource use associated with pneumonia, we also studied the minor complication of isolated acute pulmonary edema, which occurred in $5.8 \%(\mathrm{n}=363)$ of patients. This complication was associated with an $\$ 11,271 \pm 855$ increase in total hospital cost $(P<.001)$ and a $2.2 \pm 0.3$ day increase in length of stay $(P<.001)$. There was a significant, independent association between acute pulmonary edema and the development of postoperative pneumonia (OR, 3.1; CI, 2.4-4.0; $P<.001)$.

When analyzed by number of complications per patient, there was a stepwise association between number of complications and both total hospital costs and length of hospitalization $(P<.001)$ (Figure 1$)$. Patients who had 1 complication during their hospitalization had a median 
TABLE 3. Incremental median net increase in hospital cost and length of stay by complication category

\begin{tabular}{|c|c|c|c|c|c|c|c|}
\hline Complication category & $\begin{array}{c}\text { Median net increase } \\
\text { in hospital cost }(\$)\end{array}$ & $95 \%$ CI & $\begin{array}{c}P \\
\text { value }\end{array}$ & $\begin{array}{c}\text { Median net } \\
\text { increase in LOS }(d)\end{array}$ & $95 \%$ CI & $\begin{array}{c}P \\
\text { value }\end{array}$ & $\begin{array}{c}\text { Incremental cost } \\
\text { incurred annually }(\$)\end{array}$ \\
\hline Pneumonia & 29,692 & $27,590-31,793$ & $<.001$ & 10.2 & $9.7-10.7$ & $<.001$ & 23.9-27.5 million \\
\hline Sepsis & 22,404 & $21,203-23,604$ & $<.001$ & 7.0 & $6.7-7.3$ & $<.001$ & 13.8-15.4 million \\
\hline Renal failure requiring hemodialysis & 28,336 & $25,759-30,913$ & $<.001$ & 8.7 & $8.0-9.4$ & $<.001$ & 14.4-17.2 million \\
\hline Acute myocardial infarction & 20,576 & $17,672-23,480$ & $<.001$ & 3.5 & $2.8-4.2$ & $<.001$ & 7.6-10.1 million \\
\hline Stroke & 21,477 & $17,498-25,456$ & $<.001$ & 9.7 & $8.5-10.8$ & $<.001$ & 6.3-9.2 million \\
\hline Gastrointestinal bleed & 12,230 & $8,184-16,277$ & $<.001$ & 6.1 & $4.9-7.3$ & $<.001$ & $2.5-4.9$ million \\
\hline Venous thromboembolism & 25,896 & $20,934-30,859$ & $<.001$ & 7.3 & $6.0-8.5$ & $<.001$ & 2.6-3.8 million \\
\hline Cardiac tamponade & 56,547 & $44,328-68,765$ & $<.001$ & 19.3 & $16.2-22.3$ & $<.001$ & $0.9-1.4$ million \\
\hline
\end{tabular}

8-day increase in length of stay and total hospital costs increased by a median value of $\$ 21,000$. From 1 to 2 complications, there was a similar increase of 6 hospital days and $\$ 23,964$. From 2 to 3 complications, the length of hospitalization increased by 12.5 days, and the cost of hospitalization increased by an additional $\$ 35,330$.

There was a significant association between the discharge disposition and the occurrence of 1 or more complications during the index hospitalization $(P<.001)$ (Table 4$)$. Among patients with no complications, $52.5 \%(\mathrm{n}=2729)$ were discharged home as a routine discharge, whereas only $27.0 \%$ $(n=298)$ of such discharges occurred in patients with 1 or more complication. In addition, patients with no complications were more likely to use home health care $(32.8 \%$, $\mathrm{n}=1703)$ than patients with complications $(29.1 \%$, $\mathrm{n}=321)$. However, patients with complications were more likely to be discharged to skilled nursing facilities or intermediate care facilities $(29.1 \%, \mathrm{n}=321)$ than patients without complications $(11.7 \%, \mathrm{n}=608)$.

Appendix 2 summarizes outcomes of all mitral valve procedures in the NIS registry during the study period and

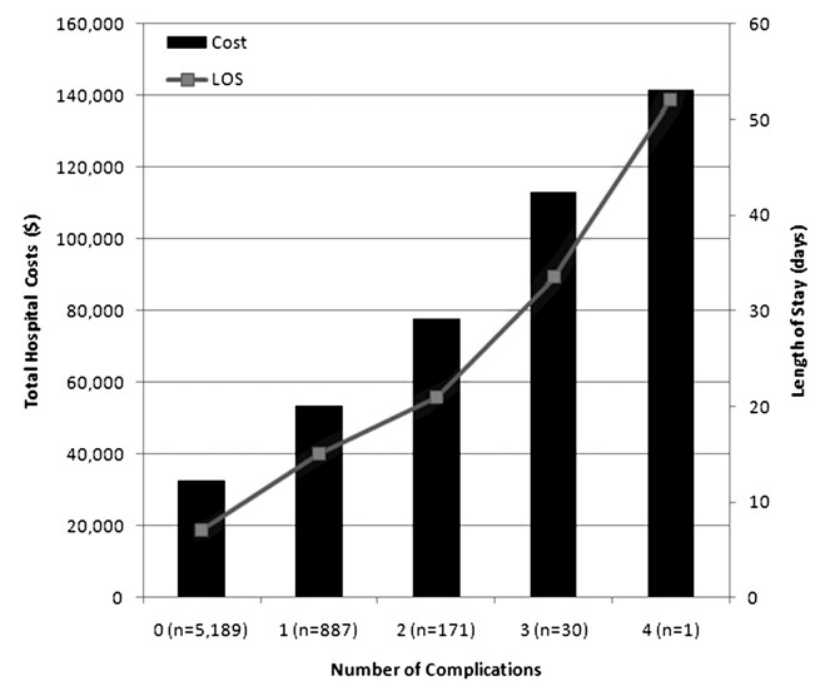

FIGURE 1. Multiplicative effect of complications on total hospital costs and length of hospital stay. LOS, Length of stay. associated lengths of stay, total hospital costs, and frequency of in-hospital complications. The 2 procedures associated with the highest median length of stay included tissue mitral valve replacement (MVR) $+\mathrm{CABG}+$ aortic valve replacement (AVR) and tissue MVR $+\mathrm{CABG}+\mathrm{AVR}+$ tricuspid valve replacement at 14 hospital days each. The median total hospital costs of all cases was $\$ 42,307$, with the highest cost occurring for tissue MVR + CABG + AVR $(\$ 66,683)$, mechanical MVR + other $(\$ 54,100)$, and tissue MVR+AVR $(\$ 54,646)$. The highest frequency of complications occurred in mitral valve repair + CABG $(7.7 \%$, $\mathrm{n}=1290$ ).

\section{DISCUSSION}

This analysis describes major complications in a national sample of patients undergoing isolated MVS and the effect of these complications on clinical outcomes and resource use. On the basis of findings in this analysis, complications related to MVS have a significant adverse impact on both clinical outcomes and resource use.

In the current healthcare environment, there is increasing pressure to improve outcomes while containing costs. ${ }^{10,11}$ Quality measures will influence how healthcare services are reimbursed in the future. In 2007, the Centers for Medicaid and Medicare Services (CMS) determined that it would no longer pay for "preventable" complications. These never events include, among others, retained foreign bodies, mediastinitis after CABG, line infections, pressure ulcers, and air embolism.

In 2011, the CMS issued the Hospital Inpatient ValueBased Purchasing Program Final Rule. Under this program, the CMS will provide higher payments to hospitals that perform well on certain quality measures relating to both clinical process and patient experience of care. This initiative is intended to help reduce health care costs and reward hospitals for the quality of care they provide to Medicare beneficiaries. ${ }^{12}$ This program will apply to hospital discharges occurring on or after October 1, 2012, with payments based on whether a hospital meets or exceeds performance standards. Defined changes in payer strategies such as these further highlight the need to better understand processes of care and how to improve them. 
TABLE 4. Discharge disposition by occurrence of an index hospitalization complication

\begin{tabular}{lccc}
\hline \multicolumn{1}{c}{ Discharge location } & $\begin{array}{c}\text { No } \\
\text { complications }\end{array}$ & $\begin{array}{c}\text { Any } \\
\text { complication }\end{array}$ & $\begin{array}{c}\boldsymbol{P} \\
\text { value }\end{array}$ \\
\hline Routine home discharge & $2729(52.5 \%)$ & $298(27.0 \%)$ & $<.001$ \\
Home health care & $1703(32.8 \%)$ & $321(29.1 \%)$ & \\
SNF, ICF, other & $608(11.7 \%)$ & $321(29.1 \%)$ & \\
Died & $121(2.3 \%)$ & $132(12.0 \%)$ & \\
Transfer to short-term hospital & $20(0.38 \%)$ & $16(1.5 \%)$ & \\
Unknown & $10(0.19 \%)$ & $13(1.2 \%)$ & \\
Against medical advice & $6(0.12 \%)$ & $1(0.09 \%)$ & \\
Total & 5197 & 1102 & \\
\hline$S N F$ Skillo & & &
\end{tabular}

$S N F$, Skilled nursing facility; $I C F$, intermediate care facility.

\section{Comorbidities}

In this current analysis of patients undergoing isolated MVS in the United States over a 2-year period, a large proportion of patients presenting for mitral surgery had significant comorbidities, as suggested by previous authors. ${ }^{5}$ Peripheral vascular disease and heart failure were present in $38 \%$ of patients undergoing surgery, and chronic obstructive pulmonary disease was present in $16.9 \%$. With the aging US population, these frequencies are likely to increase in the future; however, the demand for quality assurance and low rates of complications will grow. ${ }^{13}$

\section{Outcomes}

With an overall national in-hospital mortality rate of $4 \%$, this analysis highlights that, despite its complexity, MVS can be performed safely. Likewise, no individual complication occurred in more than $6 \%$ of patients. Nevertheless, approximately $15 \%$ of patients experienced at least 1 of the 8 complications. In-hospital mortality in the subset of patients in our analysis undergoing elective surgery was similar to that reported in the most recent analysis of isolated MVS from the Society of Thoracic Surgeons (STS) database: $2.3 \%$ versus $1.8 \%$ in the STS database. It is not surprising that the overall rates of morbidity reported in our analysis of the NIS are slightly higher than that reported in the most recent STS analysis because we included both elective and urgent cases. When isolated to elective cases, stroke was similar: $1.02 \%$ versus $1.27 \%$ in the STS database, and renal failure was similar: $2.0 \%$ versus $2.98 \%$ in the STS database. ${ }^{14}$

\section{Complications}

Among patients undergoing isolated MVS, pneumonia was the most common complication occurring in more than 1 in 20 patients, and was associated with the second largest risk-adjusted median net increase in hospital cost and length of stay. Pneumonia also resulted in the single greatest total expenditure, at approximately \$25 million annually. Sepsis and renal failure requiring dialysis, which were comparable in their effect on hospital cost and length of stay, resulted in the second and third greatest total expenditure, respectively. Of note, acute postoperative pulmonary edema had a significant impact on total hospital costs of approximately $\$ 11,000$, and there was a significant association between postoperative pulmonary edema and development of pneumonia, emphasizing the impact of perioperative management on postoperative resource use. These findings support recent efforts dedicated to providing more aggressive respiratory care and to lowering rates of infection through quality improvement initiatives aimed at formalizing process-of-care measures. ${ }^{15,16}$ In addition, our results are supported by a recent analysis of the STS database demonstrating an increase in the incidence of prolonged ventilation and pneumonia. ${ }^{5}$ Of note, cardiac tamponade had the largest effect on total cost and length of stay, but it had the lowest prevalence in our series.

In addition to the effect of specific complications on resource use, this analysis also demonstrates the multiplicative effect of complications on total costs and length of stay. Although there was a $\$ 21,000$ difference in total cost and an 8-day difference in length of hospitalization between patients who had no complications and patients who had 1 complication, the difference increased to $\$ 44,964$ and 14 days when the comparison was made against patients with 2 complications. Thus, 2 complications added $\$ 44,964$ to the total cost of hospitalization, which is greater than the median cost of admission at $\$ 35,446$.

In total, these 8 complications were annually associated with an additional $\$ 71.9$ to $\$ 89.4$ million in costs and an additional 24,000 to 29,000 inpatient hospital days during the index hospitalization alone. Although complications cannot be avoided in many cases, these data highlight the significant resources consumed when a patient experiences complications postoperatively and demonstrate areas where quality improvement initiatives may be directed to further improve cost-effectiveness and patient outcomes.

\section{Discharge Disposition}

The time horizon of this analysis is limited to outcomes during the index hospitalization. However, available discharge disposition data suggest that complications have an important impact on longer-term resource costs, both direct and indirect. There was a significant correlation between the discharge location and the presence of any complication during the index hospitalization. Among patients experiencing no complications, $25.5 \%$ more patients underwent routine home discharge compared with patients who had 1 or more complication. In addition, patients who had 1 or more complication were $17.4 \%$ more likely to be discharged to a skilled nursing facility or intermediate care facility, which clearly adds to long-term costs. These findings highlight the importance of pursuing future studies that explore the relationship between clinical outcomes and quality of life, as well as the economic effects, 
including direct cost and indirect costs, such as lost worker productivity. Furthermore, these findings suggest that providers should anticipate that patients who experience complications are more likely to require additional care after hospital discharge.

Therefore, further research that examines both hospital charges and subsequent costs outside of standard home discharge on the total economic burden of complications is necessary. Although the NIS does not contain costing data beyond the index hospitalization, there are other existing data sources that may allow for better characterization of intermediate and longer-term costs. For example, Thompson-Reuters' Marketscan Data, ${ }^{17}$ which include 43 million covered lives, capture longitudinal data related to inpatient and outpatient costs, as well as worker productivity data. The University Health System Consortium data contain detailed costing data from $90 \%$ of the academic centers nationally and have the potential to be crossmatched with detailed clinical data. Finally, physician-initiated statebased quality groups, such as the Virginia Cardiac Surgery Quality Initiative, contain uniquely detailed clinical and economic data.

\section{Limitations}

There are several limitations to our analysis. First, the NIS is an administrative database and does not contain detailed clinical information on a variety of measures, including the severity of mitral valve disease and history of cardiac operations. Second, only $20 \%$ of nationwide institutions are sampled within the NIS; therefore, there exists the potential for limitations in the number of hospitals surveyed that perform comprehensive cardiac surgery. Third, follow-up did not extend beyond the index hospitalization; consequently, important events or complications that may have occurred after discharge were not captured. Fourth, the use of ICD-9 codes to identify clinical outcomes is imperfect. There exists the potential for heterogeneity in reporting of adverse events among hospitals because data on complications were extracted from ICD-9 codes rather than protocol-defined end points as one would use in a prospective study. Such variability in reporting carries the potential to introduce bias in outcome measures. However, use of ICD-9 codes to assess clinical outcomes in administrative registries is a well-established technique. ${ }^{7}$ In a series of recent reports from the Complications Screening Project, the validity of ICD-9-CM coding of several medical and surgical complications was investigated. The coding of surgical complications was found to be clinically valid. When comparing ICD-9-CM coding and the clinical record, the positive predictive value for complications varied from $84.2 \%$ to $96.8 \%$ with kappa scores of 0.69 to $0.88{ }^{18,19}$ Furthermore, some important complications, such as postoperative atrial fibrillation and sternal wound infection, could not be identified. Finally, cost-to-charge ratios used in the analysis, and thus reported costs, may be subject to bias because variability exists in state reporting of cost-to-charge ratios (eg, variability in individual participating institutional ratios vs aggregate state ratios). Nevertheless, many of the limitations in this analysis must be understood in the context of the strengths of the NIS, which include its large size, representative quality, and standardized methodology of survey.

\section{Implications}

Although instituting standardized process-of-care measures requires a significant institutional commitment, the long-term benefits with regard to clinical outcomes and resource use cannot be underestimated. In addition, with new policy measures such as the Hospital Inpatient Value-Based Purchasing Initiative, hospitals will be placed under significant pressure to avoid such adverse events. Complications have a significant and multiplicative effect on resource use for index hospitalization. Complications also influence long-term costs based on increased need for skilled nursing and intermediate care placement on discharge, as well as lost worker productivity. Although complications can never be completely eliminated, pneumonia and sepsis, for example, represent events that may benefit from efforts at increased standardization of institutional process-of-care measures to reduce these potentially preventable complications.

In an era of increased national attention to cost effectiveness and increased patient awareness of hospital reporting of risk-adjusted outcomes, the current analysis not only shows the impact of complications on resource use but also serves as a starting point for quantifying the effect of such complications and identifying areas of future improvement and study.

\section{CONCLUSIONS}

In patients undergoing isolated MVS, postoperative complications were associated with significant increases in mortality, hospital costs, and length of stay. Among the 8 postoperative complications captured in this analysis, pneumonia resulted in the greatest total additional cost across the entire population of patients undergoing MVS, followed by renal failure requiring dialysis and sepsis. Further, stacking complications disproportionately increased the related costs. In an era with growing national attention to improving quality and containing costs, it is important to understand the impact of complications on outcomes and costs. This analysis may help drive quality improvement initiatives and improve cost-effectiveness, and ultimately improve patient outcomes.

\section{References}

1. Ad N, Barnett SD, Speir AM, Massimiano PS. Institutional and national trends in isolated MVS over the past decade. Curr Opin Cardiol. 2008;23:99-104. 
2. Barnett SD, Ad N. Surgery for aortic and mitral valve disease in the United States: a trend of change in surgical practice between 1998 and 2005. J Thorac Cardiovasc Surg. 2009;137:1422-9.

3. Gammie JS, Sheng S, Griffith BP, Peterson ED, Rankin JS, O'Brien SM, et al. Trends in MVS in the United States: results from the Society of Thoracic Surgeons Adult Cardiac Surgery Database. Ann Thorac Surg. 2009;87: 1431-7.

4. Thourani VH, Weintraub WS, Guyton RA, Jones EL, Williams WH, Elkabbani S, et al. Outcomes and long-term survival for patients undergoing mitral valve repair versus replacement: effect of age and concomitant coronary artery bypass grafting. Circulation. 2003;108:298-304.

5. Lee R, Li S, Rankin JS, O'Brien SM, Gammie JS, Peterson ED, et al. Fifteen-year outcome trends for valve surgery in North America. Ann Thorac Surg. 2011;91: 677-84.

6. Dimick JB, Chen SL, Taheri PA, Henderson WG, Khuri SF, Campbell DA Jr. Hospital costs associated with surgical complications: a report from the private-sector National Surgical Quality Improvement Program. J Am Coll Surg. 2004;199:531-7.

7. Pronovost P, Garrett E, Dorman T, Jenckes M, Webb TH III, Breslow M, et al. Variations in complication rates and opportunities for improvement in quality of care for patients having abdominal aortic surgery. Langenbecks Arch Surg. 2011;386:249-56.

8. Dimick JB, Chen SL, Taheri PA, Henderson WG, Khuri SF, Campbell DA Jr. Hospital costs associated with surgical complications: a report from the private-sector National Surgical Quality Improvement Program. J Am Coll Surg. 2004;199:531-7.

9. HCUP Nationwide Inpatient Sample (NIS). Healthcare Cost and Utilization Project (HCUP). 2000-2001. Agency for Healthcare Research and Quality, Rockville, MD. Available at: www.hcup-us.ahrq.gov/nisoverview.jsp. Accessed June 2009.

10. Institute of Medicine. Crossing the Quality Chasm: A New Health System for the Twenty-first Century. Washington, DC: National Academy Press; 2001.

11. Chassin M, Galvin RW. The urgent need to improve health care quality. Institute of Medicine National Roundtable on Health Care Quality. JAMA. 1998;280: 1000-5.

12. Centers for Medicare \& Medicaid Services (CMS), HHS. Medicare program; hospital inpatient value-based purchasing program. Final rule. Fed Regist. 2011;76:26490-547.

13. Donelan K, Rogers RS, Eisenhauer A, Mort E, Agnihotri AK. Consumer comprehension of surgeon performance data for coronary bypass procedures. Ann Thorac Surg. 2011;91:1400-6.

14. Gammie JS, Zhao Y, Peterson ED, O’Brien SM, Rankin JS, Griffith BP. Less-invasive mitral valve operations: trends and outcomes from the Society of Thoracic Surgeons Adult Cardiac Surgery Database. Ann Thorac Surg. 2010;90:1401-8.

15. Pronovost PJ, Marsteller JA, Goeschel CA. Preventing bloodstream infections: a measurable national success story in quality improvement. Health Aff (Millwood). 2011;30:628-34.

16. Berenholtz SM, Pham JC, Thompson DA, Needham DM, Lubomski LH, Hyzy RC, et al. Collaborative cohort study of an intervention to reduce ventilator-associated pneumonia in the intensive care unit. Infect Control Hosp Epidemiol. 2011;32:305-14

17. Hansen LG, Chang S. Health research data for the real world: the Thomson Reuters MarketScan databases. January 2010. Available at: http://info.thomsonhealth care.com/forms/HealthResearchWPRequest. Accessed October 1, 2011.

18. Weingart SN, Iezzoni LI, Davis RB, Palmer RH, Cahalane M, Hamel MB, et al. Use of administrative data to find substandard care: validation of the complications screening program. Med Care. 2000;38:796-806.

19. Lawthers AG, McCarthy EP, Davis RB, Peterson LE, Palmer RH, Iezzoni LI. Identification of in-hospital complications from claims data. Is it valid? Med Care. 2000;38:785-95.

\section{Discussion}

Dr Richard J. Shemin (Los Angeles, Calif). I, too, want to thank the Association for the opportunity to comment on this really detailed and excellent study and presentation. The authors were able to provide me with a detailed copy of the manuscript so I have more information than was presented today.
The fact that there is a price to pay for complications is well known to this surgical community, but this study really met the goal of quantifying the cost in dollars, days, and deaths, as well as the postdischarge resource use after an isolated mitral valve surgery. As the discussion clearly stated, this comes from an administrative data set, the Nationwide Inpatient Sample (NIS). This sample is only $20 \%$ of nonfederal hospitals in 37 states and the use of ICD-9 codes, as has been stated, has major limitations and clearly does not allow a robust evaluation for risk adjustment and other analyses that we are used to from the Society of Thoracic Surgeons (STS) data base. The overall sample in this study was taken from 2 years-2006 and 2007-more than 16,788 patients. I was somewhat surprised to find that 10,000 -plus patients actually had concomitant procedures, leaving them with isolated mitral valve surgery in 6297 , and then I was also surprised to find $50 \%$ of them actually had mitral valve repairs as opposed to replacement. I am not sure we would have predicted that was the case. The inpatient hospital mortality was actually quite good, $4 \%$ and $2 \%$ in elective cases, and $82 \%$ of the patients did not have any of complications that were studied in this particular study. The complication rates also of interest did not differ between repair and tissue replacement or mechanical replacement and a single complication clearly escalated the outcomes and the end points such as mortality, length of stay, and cost, and the need for increased resources. Very interesting was the exponential increase in cost associated with multiple complications. I was really surprised to find that pneumonia and sepsis were your most frequent complications. I suspect this was because of the study design and the choice of complications that you included in the study. These potentially preventable complications can increase the cost of hospitalization by more than $\$ 30,000$. Cardiac tamponade, which was the most costly complication in the study increasing the median cost by $\$ 57,000$, only occurred in $0.1 \%$ of the patients. Therefore, I assume the ICD-9 codes were not capturing patients going back to the operating room for bleeding, the use of blood utilization, and as you mentioned the lack of studying atrial fibrillation, which is very frequent, or sternal wound infection, which is infrequent but very costly, is clearly a limitation. Therefore, I think this study only looks at the tip of the iceberg as far as the true cost of complications after isolated mitral valve surgery. Therefore, the study challenges us to really improve on the strategies that we need to incorporate to reduce the costs of taking care of our patients. Therefore, I have 4 questions for you.

First, do you have any data on the impact of surgical volume at these various hospitals, whether it be total cardiac surgery volume or mitral valve surgery volume, on the frequency of the complications, or the cost?

Dr Iribarne. Thank you, Dr Shemin, for your careful and thoughtful review of our manuscript.

First, regarding the quality of the data, NIS has a number of attractive features. It is accessible, inexpensive, and easy to use. Moreover, it contains information on the clinical and economic data of the patient, as well as information on the institution. Finally, although crude, the use of ICD-9 codes to extract clinical information from administrative datasets is a proven and increasingly applied research approach.

However, we agree that this data has all of the limitations associated with the use of large claims datasets. For example, as you 
point out, the incidence of tamponade was clearly underestimated by this analysis. Further, sepsis was likely overcoded and more likely overlaps with other types of cardiovascular compromise and pressor dependence.

Our group is exploring the use of other large data sources to pursue similar questions to those proposed here. These might include the Centers for Medicare and Medicaid Services (CMS), Thompson-Reuters' Marketscan data, the University Health System Consortium (UHSC) data, and various statewide quality initiatives. Finally, in the future, STS data may be linked to costing data, and this may provide a powerful tool for pursuing these cost questions.

With regard to the first question, it is possible to capture institutional volume data in the NIS. Other groups have done this and, in fact, we have explored questions using this data. However, for this analysis, we did not explore the relationship between volume cost. I think that this is, however, an important but complex group of questions that are worth exploring.

Dr Shemin. Clearly, if one does find that to be the case as we find in many other areas of surgery, you can incorporate and find best practices that can be taught to others as we look for ways to find solutions.

The second question has to do with whether or not there is anything in your data set that will allow you to predict the $15 \%$ of patients who would have the complications that you showed us so that strategies can be targeted to those patients and specific complications and not have a very expensive protocol to try to incorporate everyone?

Dr Iribarne. Defining the relationship between preoperative comorbidities and outcomes, including complications and cost, is possible with this data. We have previously explored this in other populations, including a single institution's series of ventricular assist device patients. We are also interested in exploring the usefulness of various severity or comorbid illness tools, including the
Deyo modification to the Charlston index, the Elixhauser index, and the 3M APR-DRG, to pursue other questions related to volume-outcome relationships across levels of patient risk and comparing disease-specific therapeutic options across various patient risk strata.

Dr Shemin. And I have a methodologic question. When you went ahead and looked at the $20 \%$ costs and then you looked at the total costs by increasing it by a factor of 5 , is that technically correct? As I understand it, there are actually correction values that come with the nationwide sample that allow you to look at the total cohort and not necessarily do some arithmetic by increasing $20 \%$ to $100 \%$ by just multiplying by 5 .

Dr Iribarne. You are correct. The NIS assigns a correction factor to each patient and institution. The cost estimates that we presented were ranges based on bootstrapped estimates. Therefore, the estimates are unlikely to differ significantly. Nevertheless, per your suggestion, the final version of the manuscript uses an NIS-provided correction factor to calculate this estimate.

Dr Shemin. The final question is, now that you have these data, what types of things do you think we need to do to reduce this complication rate and better treat these patients?

Dr Iribarne. Thank you for your question. This analysis alone does nothing to improve outcomes or decrease costs. However, we hope that it highlights opportunities for improving outcomes, decreasing costs, and therefore offering greater value in health care. As you suggested earlier, as a health care community, we need to be more aggressive by identifying best practices. We applaud these efforts, particularly of surgeon-driven statewide efforts to improve quality, including those in Northern New England, Virginia, and Washington State. We hope that other regions adopt similar methods. Further we see an opportunity for similar continuous quality improvement efforts to be based on specific therapies, such as transplantation, ventricular assist devices, and valvular therapies. 
APPENDIX 1. Univariate analysis of baseline characteristics

\begin{tabular}{|c|c|c|c|}
\hline Baseline variable & No complication & Any complication & $P$ value \\
\hline \multicolumn{4}{|l|}{ Demographics } \\
\hline Age & $56.7 \pm 0.27$ & $60.4 \pm 0.51$ & $<.001$ \\
\hline Male & $2,535(48.9 \%)$ & $527(48.4 \%)$ & .941 \\
\hline Elective procedure & $3,650(70.3 \%)$ & $453(41.6 \%)$ & $<.001$ \\
\hline Primary payer: Medicare & $1,975(38.1 \%)$ & $583(53.5 \%)$ & $<.001$ \\
\hline Primary payer: Private & $2,460(47.4 \%)$ & $321(29.5 \%)$ & $<.001$ \\
\hline \multicolumn{4}{|l|}{ Comorbidities } \\
\hline Peripheral vascular disease & $1,842(35.5 \%)$ & $564(51.8 \%)$ & $<.001$ \\
\hline Heart failure & $1,839(35.4 \%)$ & $557(51.2 \%)$ & $<.001$ \\
\hline Chronic obstructive pulmonary disease & $836(16.1 \%)$ & $228(20.9 \%)$ & $<.001$ \\
\hline History of any malignancy & $840(16.2 \%)$ & $106(9.7 \%)$ & $<.001$ \\
\hline Diabetes mellitus & $556(10.7 \%)$ & $114(10.5 \%)$ & .811 \\
\hline Chronic kidney disease & $329(6.3 \%)$ & $242(22.2 \%)$ & $<.001$ \\
\hline Cerebrovascular disease & $182(3.5 \%)$ & $195(17.9 \%)$ & $<.001$ \\
\hline Previous myocardial infarction & $195(3.8 \%)$ & $24(2.2 \%)$ & .011 \\
\hline Rheumatologic disease & $113(2.2 \%)$ & $29(2.7 \%)$ & .327 \\
\hline Diabetes with chronic complications & $50(0.96 \%)$ & $32(2.9 \%)$ & $<.001$ \\
\hline Moderate or severe liver dysfunction & $50(0.96 \%)$ & $32(2.9 \%)$ & $<.001$ \\
\hline Peptic ulcer disease & 0 & $45(4.13 \%)$ & $<.001$ \\
\hline Mild liver dysfunction & $20(0.39 \%)$ & $10(0.92 \%)$ & .020 \\
\hline Hemiplegia or paraplegia & $9(0.17 \%)$ & $14(1.3 \%)$ & .37 \\
\hline Metastatic solid tumor & $10(0.19 \%)$ & $4(0.37 \%)$ & .267 \\
\hline Human immunodeficiency virus & $7(0.13 \%)$ & $6(0.55 \%)$ & .006 \\
\hline Dementia & $2(0.04 \%)$ & 0 & .517 \\
\hline
\end{tabular}

APPENDIX 2. Length of stay, total hospitalization costs, and frequency of any in-hospital complication by procedure type

\begin{tabular}{|c|c|c|c|c|c|c|}
\hline Procedure type & Frequency & Percent & Median LOS (d) & Median total cost (\$) & In-hospital complication frequency & $\%$ \\
\hline Isolated mMVR & 1732 & 10.32 & 9 & 39,464 & 377 & 2.25 \\
\hline $\mathrm{mMVR}+\mathrm{AVR}$ & 445 & 2.65 & 11 & 50,600 & 109 & 0.65 \\
\hline $\mathrm{mMVR}+\mathrm{CABG}$ & 776 & 4.62 & 12 & 47,600 & 334 & 1.99 \\
\hline mMVR + MAZE & 531 & 3.16 & 9 & 38,491 & 65 & 0.39 \\
\hline mMVR + other & 605 & 3.6 & 13 & 54,100 & 190 & 1.13 \\
\hline Isolated $\mathrm{MVr}$ & 3283 & 19.56 & 6 & 30,967 & 359 & 2.14 \\
\hline $\mathrm{MVr}+\mathrm{AVR}$ & 637 & 3.79 & 10 & 44,926 & 156 & 0.93 \\
\hline $\mathrm{MVr}+\mathrm{CABG}$ & 2773 & 16.52 & 11 & 43,200 & 1290 & 7.68 \\
\hline $\mathrm{MVr}+\mathrm{CABG}+\mathrm{AVR}$ & 480 & 2.86 & 11 & 50,618 & 178 & 1.06 \\
\hline $\mathrm{MVr}+\mathrm{CABG}+\mathrm{MAZE}$ & 613 & 3.65 & 11 & 45,753 & 201 & 1.20 \\
\hline $\mathrm{MVr}+\mathrm{MAZE}$ & 1022 & 6.09 & 8 & 36,432 & 117 & 0.70 \\
\hline $\mathrm{MVr}+$ other & 290 & 1.73 & 10 & 47,600 & 65 & 0.39 \\
\hline Isolated tMVR & 1282 & 7.64 & 11 & 44,850 & 358 & 2.13 \\
\hline $\mathrm{tMVR}+\mathrm{AVR}$ & 374 & 2.23 & 13 & 54,646 & 118 & 0.70 \\
\hline $\mathrm{tMVR}+\mathrm{CABG}$ & 861 & 5.13 & 12 & 50,800 & 368 & 2.19 \\
\hline $\mathrm{tMVR}+\mathrm{CABG}+\mathrm{AVR}$ & 250 & 1.49 & 14 & 66,683 & 88 & 0.52 \\
\hline $\mathrm{tMVR}+\mathrm{CABG}+\mathrm{AVR}+\mathrm{TVR}$ & 3 & 0.02 & 14 & 49,000 & 1 & 0.01 \\
\hline tMVR + MAZE & 435 & 2.59 & 10 & 43,228 & 76 & 0.45 \\
\hline tMVR + other & 396 & 2.36 & 12 & 54,047 & 121 & 0.72 \\
\hline
\end{tabular}

Total procedures $=16,788$

$M V r$, Mitral valve replacement; $m M V R$, mechanical mitral valve replacement; $t M V R$, tissue mitral valve replacement; $T V R$, tricuspid valve replacement; $C A B G$, coronary artery bypass grafting; $L O S$, length of stay; $A V R$, aortic valve replacement. All percentages reported use total procedures $(\mathrm{n}=16,788)$ as the denominator. 